\title{
The Predictive Value of the Neutrophil-to-Lymphocyte and Platelet-to-Lymphocyte Ratio in Patients with Recurrent Idiopathic Granulomatous Mastitis
}

\author{
Sami Akbulut (1D), Tevfik Tolga Şahin (1) \\ Department of Surgery and Liver Transplant Institute, İnönü University School of Medicine, Malatya, Turkey
}

Cite this articles as: Akbulut S, Şahin TT. The Predictive Value of the Neutrophil-to-Lymphocyte and Platelet-to-Lymphocyte Ratio in Patients with Recurrent Idiopathic Granulomatous Mastitis. Eur J Breast Health 2020; 16(3): 226-227.

Dear Editor,

We read the recent article "The predictive value of the neutrophil-to-lymphocyte and platelet-to-lymphocyte ratio in patients with recurrent idiopathic granulomatous mastitis " published by Cetinkaya et al. (1) with great interest. Authors stated that they investigated the relationship between the neutrophil-to-lymphocyte ratio (NLR), platelet-to-lymphocyte ratio (PLR) and the prognosis of idiopathic granulomatous mastitis (IGM).

We do not agree with the authors' statement which indicated that although the etiology of IGM was unclear, tuberculosis (TB), sarcoidosis, mycotic, and parasitic infections may play a role in the development of the IGM. It is a common fact that granulomatous mastitis (GM) is classified into two according to the underlying etiology; primary (idiopathic) and secondary (specific) GM. Secondary GM is caused by various specific infectious (bacteria, fungi, parasites) and non-infectious diseases (sarcoidosis, Wegener's granulomatosis, etc.). On the other hand, IGM is characterized by chronic non-caseification (non-necrotizing) granulomatous inflammation which does not have a specific underlying disease. TB should be included in the differential diagnosis in the endemic areas such as Turkey. Besides, TB and other infections have no direct relationship with IGM.

The authors have shown that patients with preoperative NLR $\geq 5.02$ have 9.33 times higher risk of recurrence when compared to patients with NLR<5.02 ( $\mathrm{p}=0.013)$. We have analyzed this result and calculated the categorical correlation coefficient for this relationship and the Phi coefficient $(\varnothing)$ was calculated as $0.437(\mathrm{p}=0.005)$. This shows a positive and strong relationship between the high NLR and the risk of recurrence. The authors have shown that the sensitivity and specificity of high NLR for prediction of recurrences were $62.5 \%$ and $84.8 \%$; respectively. The most important part of this analysis is the negative predictive value of high NLR for prediction of recurrence to be $90.3 \%$. In other words, high NLR gives a better idea regarding patients that will not develop recurrence rather than predicting the recurrence of IGM. This theory is supported by the fact that high NLR had a specificity of $84.8 \%$ and a positive predictive value of $50.2 \%$.

The authors should have performed a multivariate analysis to determine the independent risk factors for recurrence by using the variables with a p-value $<0.1$ that was determined in the univariate analysis. However, the authors did not perform any logistic regression analysis. We have used the methodology on the variables expressed in authors' Table- 2 and performed a logistic regression analysis. The only variable that fit the criteria was NLR and the multivariate analysis yielded similar results as the univariate analysis. We were able to show that NLR $\geq 5.02$ was an independent risk factor determining the recurrence in patients with IGM (Logistic regression with backward LR; Wald: 6.49, $\mathrm{p}=0.011, \mathrm{OR}=9.3395 \% \mathrm{CI}=1.67-52.06)$.

Recently, NLR is used as a surrogate marker for the systemic inflammatory process. During systemic inflammation, neutrophil counts increase, and lymphocyte counts decrease which increases NLR. For this reason. NLR can be used to predict the severity of the disease in various clinical conditions such as various infectious diseases, cancer, and critically ill patients. In literature, there is only one study 
analyzing the role of NLR in IGM. Kargin et al. (2) have compared NLR in patients with $(n=7)$ and without $(n=52)$ recurrence and have found that NLR was significantly higher in patients with a recurrence $(\mathrm{p}<0.001)$ but they have not performed a ROC analysis to support their observations.

\section{References}

1. Çetinkaya ÖA, Çelik SU, Terzioğlu SG, Eroğlu A. The Predictive Value of the Neutrophil-to-Lymphocyte and Platelet-to-Lymphocyte Ratio in Patients with Recurrent Idiopathic Granulomatous Mastitis. Eur J Breast Health 2020; 16: 61-65. (PMID: 31912016) [Crossref]

2. Kargin S, Turan E, Esen HH, Kargin NC. Role of Pre-treatment Neutrophil-Lymphocyte Ratio in the Prediction of Recurrences After Granulomatous Mastitis Treatment. Turkiye Klinikleri J Med Sci 2020; 40: 46-51.

\section{Author's Reply}

\section{Re: The Predictive Value of the Neutrophil-to-Lymphocyte and Platelet-to-Lymphocyte Ratio in Patients with Recurrent Idiopathic Granulomatous Mastitis}

\author{
Ömer Arda Çetinkaya ${ }^{1}$ (D), Süleyman Utku Çelik ${ }^{1,2}$ (D), \\ Serdar Gökay Terzioğlu ${ }^{3}$ (D), Aydan Eroğlu ${ }^{4}$ (D) \\ ${ }^{1}$ Department of General Surgery, Ankara University School of Medicine, \\ Ankara, Turkey \\ ${ }^{2}$ Clinic of General Surgery, Gulhane Training and Research Hospital, \\ Ankara, Turkey \\ ${ }^{3}$ Clinic of General Surgery, Ankara Numune Training and Research \\ Hospital, Ankara, Turkey \\ ${ }^{4}$ Surgical Oncology Unit, Department of General Surgery, Ankara University \\ School of Medicine, Ankara, Turkey
}

\section{Dear Editor,}

We read the comment on our article (1), "The Predictive Value of the Neutrophil-to-Lymphocyte and Platelet-to-Lymphocyte Ratio in Patients with Recurrent Idiopathic Granulomatous Mastitis” with great interest.

We agree with Akbulut and Sahin. Granulomatous mastitis (GM) is a heterogeneous group of diseases of unknown etiology; however, idiopathic granulomatous mastitis (IGM) is a diagnosis of exclusion.

With a cut-off value of 5.02, the preoperative neutrophil-to-lymphocyte ratio had a sensitivity of $62.5 \%$ and a specificity of $84.8 \%$, as we reported, as well as a positive predictive value (PPV) of $50.0 \%$ and a negative predictive value (NPV) of $90.3 \%$ in predicting recurrent IGM. Sensitivity and specificity are independent of the population of interest subjected to the test. They are dependent on the cut-off value above or below which the test is positive. In other words, these diagnostic performance parameters are threshold dependent. However,
PPV and NPV are dependent on the prevalence of the disease in the population of interest and is known as changeable parameters according to prevalence (2). In rare cases such as IGM, the recurrence rate may change from region to region as well as according to treatment strategies. Thus, if the prevalence of the disease in a $2 \times 2$ table is not the same as in the population, interpreting the results according to PPV or NPV may not be the right approach (3).

Multivariate analysis is used to describe analyses of data where there are multiple variables or observations for each unit or individual and is used to further examine the variables indicated by the univariate analysis. Theoretically, every variable collected in the study could be a candidate predictor. However, to reduce the risk of false-positive findings and improve model performance, the events per variable rule of thumb is commonly applied and at a minimum set to 10 for multivariate logistic regression. This rule of thumb recommends that at least 10 individuals need to have developed the outcome of interest for every predictor variable included in the model (4). For logistic regression, the number of events is given by the size of the smallest of the outcome categories. In our study, because of relatively low number of patients and the low recurrence rate (8/33), we could not run multivariate analysis. Interestingly, we are not able to understand how Akbulut and Sahin run a multivariate analysis, without any dataset in their hands. Of course, this is impossible. Because selecting variables for regression analysis using univariate analysis is not the only way; there are multiple methods used for choosing the variables to be included in the final model without introducing bias into the analysis. These variables can be determined by the literature review, the experience in the field, correlation, or maybe risk factors for the disease. We think that to perform a multivariate analysis using the data in table 2 (1) will give a wrong direction.

Corresponding Author: Ömer Arda Çetinkaya

e-mail: omerardacetinkaya@yahoo.com

DOI: $10.5152 /$ ejbh.2020.5786

Received: 15.05 .2020

Accepted: 18.05 .2020

Cite this article as: Çetinkaya ÖA, Çelik SU, Terzioğlu SG, Eroğlu A. Re: The Predictive Value of the Neutrophil-to-Lymphocyte and Platelet-to-Lymphocyte Ratio in Patients with Recurrent Idiopathic Granulomatous Mastitis. Eur J Breast Health 2020; 16(3): 227.

\section{References}

1. Çetinkaya ÖA, Çelik SU, Terzioğlu SG, Eroğlu A. The Predictive Value of the Neutrophil-to-Lymphocyte and Platelet-to-Lymphocyte Ratio in Patients with Recurrent Idiopathic Granulomatous Mastitis. Eur J Breast Health 2020; 16: 61-65. (PMID: 31912016) [Crossref]

2. Lalkhen AG, McCluskey A. Clinical tests: sensitivity and specificity. Continuing Education in Anaesthesia Critical Care \& Pain 2008; 8: 221-223. [Crossref]

3. Molinaro AM. Diagnostic tests: how to estimate the positive predictive value. Neurooncol Pract 2015; 2: 162-166. (PMID: 31386059) [Crossref]

4. Shipe ME, Deppen SA, Farjah F, Grogan EL. Developing prediction models for clinical use using logistic regression: an overview. J Thorac Dis 2019; 11: 574-584. (PMID: 31032076) [Crossref] 